\title{
Novel modification of an artificial placenta: pumpless arteriovenous extracorporeal life support in a premature lamb model
}

\author{
Yuichiro Miura' ${ }^{\text {, Tadashi Matsuda }}{ }^{1}$, Akio Funakubo², Shinpei Watanabe ${ }^{1}$, Ryuta Kitanishi ${ }^{1}$, Masatoshi Saito \\ and Takushi Hanita ${ }^{1}$
}

BACKGROUND: Previous studies aimed at developing an artificial placenta have had limited success. We hypothesized that the introduction of a high-performance membranous oxygenator to a pumpless artificial placenta could prolong the survival time of premature lambs.

METHODS: Immediately after delivery of the fetuses, the umbilical vessels were cannulated and connected to the pumpless artificial placenta. Both the fetuses and the circuit were submerged in a warm saline bath.

RESULTS: Five fetuses survived for $18.2 \pm 3.2$ (mean \pm SEM) $h$ after attachment to the artificial placenta, which maintained fetal circulation. Circuit blood flow was positively correlated with mean arterial pressure and negatively correlated with blood lactate levels. Milrinone administration transiently decreased lactate levels, although dopamine administration unexpectedly induced a marked increase in the lactate levels despite an elevated arterial pressure and improved circuit blood flow.

CONCLUSION: We prolonged the survival of fetal lambs using a high-performance membranous oxygenator with a small priming volume. The increased systemic resistance induced by vasoconstrictors may increase the circuit blood flow excessively, resulting in circulation failure in systemic organs; therefore, vasodilators may be more useful than vasoconstrictors for maintaining organ blood flow within this circuit.

D espite progress in neonatal intensive care, effective treatments for premature low-birth-weight infants with cardiopulmonary abnormalities are lacking. The use of an artificial placenta has been proposed, but previous studies have had limited success, possibly because the circuit volume was too large and the resistance of the circuit was too high (1-5). A more compact circuit may be a solution; in 2009, Reoma et al. reported a 4-h survival period using a pumpless artificial placenta for the first time (6). However, the fetuses could not survive for longer than $4 \mathrm{~h}$ because of fetal circulatory deterioration. The authors concluded that prolonging the survival time any further was difficult without the assistance of a roller pump.
Is it impossible to prolong the survival time using a pumpless artificial placenta? The goal of our study was to prolong survival time by improving the following two points. First, we developed a high-performance membranous oxygenator jointly with the group of A. Funakubo, who is a pioneer in the development of the membranous oxygenator $(7,8)$. Our membranous oxygenator enabled a reduction in priming volume of $40 \%$, as compared with that used in Reoma's experiment (6), while maintaining membrane surface area. Second, we administered a vasodilator to the fetuses to maintain circulation in systemic organs.

\section{RESULTS}

Table 1 compares the values obtained in Reoma's experiments (6) with those obtained in our study. The fetuses in our experimental group had a shorter gestational age, a lower birth weight, and a longer survival time than those in Reoma's experiment. In this study, five fetuses were successfully connected to the artificial placenta, and fetal circulation was maintained for $18.2 \pm 3.2 \mathrm{~h}$ (each fetus survived for 14.0, 29.8, 18.2, 11.3 , and $17.5 \mathrm{~h}$, respectively). Fetal death was not caused by infection or the deterioration of the membranous oxygenator in any of the cases, but rather by heart pump failure as a result of hyperlactatemia arising from peripheral circulation failure. Spasms of the umbilical vessels were not observed, probably because of the preventive effect of continuous infusion of lipoprostaglandin $\mathrm{E}_{1}$ during the experiments.

Figure 1 shows the changes in physiologic parameters over time in the longest survivor. At $4 \mathrm{~h}$ after birth, a significant decrease in circuit blood flow, to $\sim 15 \%$ of normal sheep placental blood flow (9), and a marked increase in blood lactate level were observed; gas exchange in the membranous oxygenator was maintained. We administered dopamine to the fetus to increase circuit blood flow via an inotropic cardiac effect and vasoconstriction, but administration had to be discontinued $4 \mathrm{~h}$ later because of a further increase in blood lactate level. After transfusion with a red blood cell concentrate for volume expansion, we next administered milrinone 
Table 1. Comparison between the results of Reoma's experiment (6) and this study

Experimental group
Reoma's
experiment

Figure 1. Changes in physiologic parameters over time in the longest survivor. The black solid lines show mean arterial pressure (MAP) $(\mathrm{mmHg})$, the gray solid lines show circuit blood flow $\left(\mathrm{ml} \cdot \mathrm{kg}^{-1} \cdot \mathrm{min}^{-1}\right)$, the triangles show $\mathrm{O}_{2}$ content $(\mathrm{g} / \mathrm{dl})$, the circles show $\mathrm{P}_{\text {CO2 }}$ (torr), and the diamonds show blood lactate level $(\mathrm{mg} / \mathrm{dl})$. Only the lactate level uses the right scale bar. Low-molecular-weight heparin and lipo-prostaglandin $\mathrm{E}_{1}$ were administered continuously to the fetus during the experiment. DOA, dopamine; $\mathrm{MLN}$, milrinone; RCC, red blood cell concentration.

(MLN) to induce an inotropic cardiac effect and vasodilation, thereby increasing circuit blood flow. Circuit blood flow increased simultaneously with mean arterial pressure (MAP), followed by a decrease in blood lactate level for a period of $4 \mathrm{~h}$ after the start of MLN administration. However, blood lactate levels gradually began to increase, and it became impossible to sustain life.

Figure 2a shows the correlation between circuit blood flow and MAP in the five fetuses. The Spearman correlation coefficient was 0.718 ( $P$ value $<0.01)$. Figure $2 \mathrm{~b}$ shows the correlation between circuit blood flow and blood lactate levels in the five fetuses. The Spearman correlation coefficient was -0.717 $(P$ value $<0.01)$. The Spearman correlation coefficients for the other parameters (circuit blood flow and $\mathrm{O}_{2}$ content, circuit blood flow and $\mathrm{P}_{\mathrm{CO}_{2}}$, MAP and blood lactate levels, MAP and $\mathrm{O}_{2}$ content, and blood lactate levels and $\mathrm{P}_{\mathrm{CO} 2}$ ) were $<0.7$.
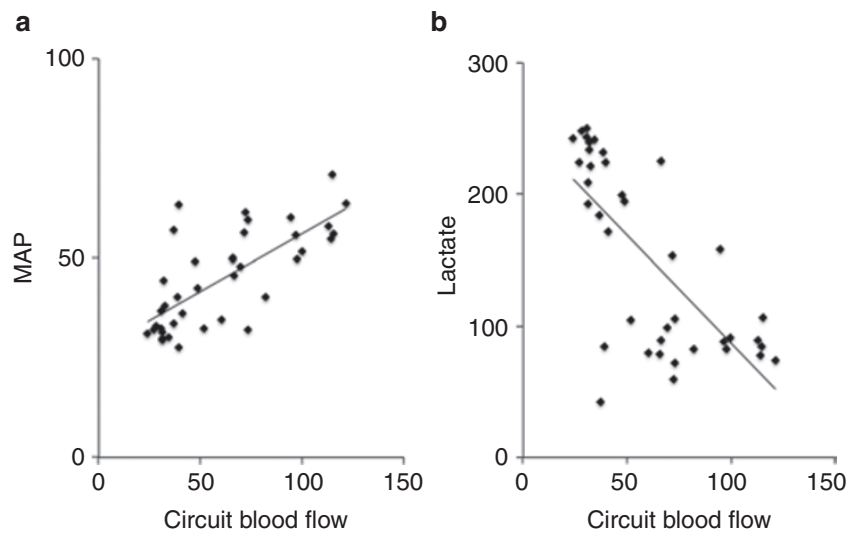

Figure 2. Scatter diagrams of parameters judged to be correlated. (a) Correlation between circuit blood flow $\left(\mathrm{ml}^{\prime} \mathrm{kg}^{-1} \cdot \mathrm{min}^{-1}\right)$ and mean arterial pressure (MAP) ( $\mathrm{mmHg}$ ). The Spearman correlation coefficient is 0.718 ( $P$ value $<0.01)$. (b) Correlation between the circuit blood flow $\left(\mathrm{ml} \cdot \mathrm{kg}^{-1} \cdot \mathrm{min}^{-1}\right)$ and the blood lactate levels $(\mathrm{mg} / \mathrm{dl})$. The Spearman correlation coefficient is -0.717 ( $P$ value $<0.01$ ). Circuit blood flow was apparently correlated with both MAP and blood lactate levels.

Table 2. Ratio of blood flow in fetal organs

\begin{tabular}{lc} 
& Mean \pm SEM \\
\hline Cerebral gray matter & $0.63 \pm 0.04^{*}$ \\
Cerebral white matter & $0.70 \pm 0.03^{*}$ \\
Medulla oblongata & $0.61 \pm 0.04^{*}$ \\
Left ventricle & $1.38 \pm 0.27$ \\
Right ventricle & $1.34 \pm 0.29$ \\
Left lung & $1.01 \pm 0.69$ \\
Right lung & $0.98 \pm 0.58$ \\
Adrenal gland & $1.29 \pm 0.19$ \\
Liver & $1.08 \pm 0.21$ \\
Kidney & $0.85 \pm 0.22$ \\
\hline
\end{tabular}

Each numerical value represents the relative value that was obtained by dividing the value for the organ blood flow obtained after birth by that obtained before birth.

${ }^{*} P<0.05$ for the values obtained before birth vs. the values obtained after birth.

Table 3. Performance of the membranous oxygenator

\begin{tabular}{lcc}
\hline & 1-6h after birth & 6-24 $\mathrm{h}$ after birth \\
\hline $\mathrm{O}_{2}$ transfer rate $(\mathrm{ml} / \mathrm{min})$ & $10.5 \pm 1.2$ & $12.2 \pm 1.0$ \\
$\mathrm{CO}_{2}$ transfer rate $(\mathrm{ml} / \mathrm{min})$ & $11.6 \pm 2.5$ & $13.7 \pm 2.0$ \\
\hline
\end{tabular}

All values are expressed as mean \pm SEM.

Circuit blood flow was apparently correlated with both MAP and blood lactate levels.

Table 2 shows the ratio of organ blood flow measured using the colored microsphere technique. Blood flow to the cerebral gray matter $(P$ value $<0.05)$, cerebral white matter $(P$ value $<$ $0.05)$, and medulla oblongata $(P$ value $<0.05)$ decreased significantly, whereas no significant changes in blood flow to both lungs and the other tissues were observed.

Table 3 shows the performance of the membranous oxygenator. No significant change in the gas transfer rate for either $\mathrm{O}_{2}$ or $\mathrm{CO}_{2}$ was observed between the two times. 


\section{DISCUSSION}

In this study, we made three important observations. First, the survival times of the fetuses were prolonged from $3.5 \pm 0.4 \mathrm{~h}$ in Reoma's study (6) to $18.2 \pm 3.2 \mathrm{~h}(n=5)$ in our experiment. The maximal survival period was $29.8 \mathrm{~h}$. All five fetuses in our experiment survived for more than $11 \mathrm{~h}$ attached to the artificial placenta, which maintained fetal circulation. The absence of a significant increase in blood flow in the lungs was considered to be evidence of the maintenance of fetal circulation.

Second, a membranous oxygenator with a smaller priming volume may be capable of supporting smaller fetuses. The fetuses in our experimental group had a shorter gestational age, a lower birth weight, and a longer survival time than those in Reoma's experiment (6), as shown in Table 1. These parameters showed significant differences between the two groups. In addition, our artificial placenta had a smaller priming volume than that used in Reoma's experiment. The smaller priming volume reduced the preload of the fetal heart, enabling the fetal circulation to be maintained for a longer time than in Reoma's experiment. As the reported placental blood volume of the sheep was $23.1-48.1 \mathrm{ml} / \mathrm{kg}$ (10), the appropriate blood volume for our fetuses were estimated to be $67-140 \mathrm{ml}$. Therefore, the circuit volume of our artificial placenta $(60 \mathrm{ml})$ may be sufficiently low. However, the resistance of our membranous oxygenator $\left(0.85 \mathrm{mmHg} \cdot \mathrm{min} \cdot \mathrm{kg} \cdot \mathrm{ml}^{-1}\right)$ may still be too high, as the placental resistance of sheep was previously reported to be $0.14-0.30 \mathrm{mmHg} \cdot \mathrm{min} \cdot \mathrm{kg} \cdot \mathrm{ml}^{-1}(9,11,12)$. If an artificial placenta with a lower resistance can be developed, such a device may further prolong fetal survival time by reducing afterload to the fetal heart.

Third, vasodilators may be more useful than vasoconstrictors for maintaining organ blood flow, especially to the brain, and driving the pumpless artificial placenta. As shown in Figure 2, circuit blood flow was apparently correlated with both MAP and blood lactate levels. Consequently, it might be possible to maintain circuit blood flow and blood lactate levels simultaneously by optimally regulating MAP. However, dopamine administration unexpectedly induced a marked increase in blood lactate levels despite an elevated MAP and improved circuit blood flow (Figure 1). The increased systemic resistance induced by dopamine might excessively increase circuit blood flow, thereby resulting in circulation failure in the systemic organs and a decrease in blood flow in cerebral gray matter, cerebral white matter, and medulla oblongata, as shown in Table 2. Therefore, the use of a vasoconstrictor may be undesirable in such situations. However, the blood lactate level decreased transiently after the start of MLN administration (Figure 1). Although this result suggests that vasodilators may be more useful than vasoconstrictors for maintaining organ blood flow, the actual organ blood flow could not be measured during the period of MLN administration in our study. In our next experiments, we plan to administer MLN at the start of the experiment in the hope of prolonging the survival period and improving organ blood flow. Better circulatory management may further prolong the survival period, as no significant deterioration in the performance of the membranous oxygenator was observed, as shown in Table 3.

In conclusion, we were able to prolong the survival period from $3.5 \pm 0.4 \mathrm{~h}$, as reported in Reoma's study (6), to $18.2 \pm$ $3.2 \mathrm{~h}$ in our experiment, demonstrating the possible usefulness of a pumpless artificial placenta. However, this result remains insufficient for practical use. Therefore, further improvements of the circuit and of circulatory management are needed.

\section{METHODS}

\section{Animal Preparation}

With the approval of the Animal Care and Use Committee of the Tohoku University School of Medicine (20HpA-3, 21MdA-4, 22MdA18), this study was carried out from September 2009 to March 2011. The preparations for this experiment were very similar to those used in our previous studies (13-16). In brief, a total of five Suffolk ewes with timed pregnancies underwent surgery between 120 and $130 \mathrm{~d}$ of gestation. The ewes were intubated, ventilated, and anesthetized with $1.5 \%-2 \%$ isoflurane during all the procedures. After a maternal laparotomy and hysterotomy, polyvinyl catheters were placed in the fetal superior vena cava, inferior vena cava, distal abdominal aorta, and amniotic cavity. All the catheters were exteriorized through a small incision in the flank of each ewe. After surgery, the ewes were unrestrained and housed in individual cages, with free access to water and food throughout the study period. A recovery period of $5 \mathrm{~d}$ was allowed before the start of the experiments; during that period, appropriate antibiotics were administered to the mother, fetus, and amniotic cavity.

\section{Experimental Protocol}

Blood flow in the fetal organs was measured using the colored microsphere technique on the day before the cesarean section. A cesarean section was performed $5 \mathrm{~d}$ after the initial operation. The ewes were intubated, ventilated, and anesthetized with $1.5 \%-2 \%$ isoflurane during all the procedures. After delivery of the fetuses, one polyvinyl cannula (8-10 French, Duraflo II; Edwards Lifesciences, Irvine, CA) was placed into the umbilical artery, and one polyvinyl cannula (12-14 French, Argyle Trocar catheter; Covidien, Dublin, Ireland) was placed into the umbilical vein; the fetuses were then connected to the artificial placenta. The tip of the arterial cannula was placed at a distance of $10 \mathrm{~cm}$ from the umbilical ring in the umbilical artery and did not reach the abdominal aorta. The tip of the venous cannula was placed at a distance of $5 \mathrm{~cm}$ from the umbilical ring in the umbilical vein in the round ligament before the ductus venosus. Immediately after the umbilical cord was cut, the fetuses were submerged in a saline bath warmed to $39^{\circ} \mathrm{C}$. Echocardiography was performed to confirm that the direction of the flow through the ductus arteriosus was right to left. The fetuses were continuously treated with low-molecularweight heparin to prevent blood coagulation and lipo-prostaglandin $\mathrm{E}_{1}$ to prevent closure of the ductus arteriosus. The initial dose of lowmolecular-weight heparin was 100 units per hour, with appropriate adjustments thereafter, and the initial dose of lipo-prostaglandin $E_{1}$ was $1.0 \mu \mathrm{g}$ per hour and the dosage was increased when echocardiography showed signs of ductal closure. The dosages of both drugs were calculated according to the clinical dosages and fetal body weights. Blood flow in the fetal organs was measured once again within 2-12 h after birth using the microsphere technique.

\section{Circuit}

Figure 3 shows the schema of our artificial placenta. The circuit was simply composed of three main components-an outflow tube, a prototype membranous oxygenator, and an inflow tube. Polyvinyl tubes (1/4 inch in diameter and $20 \mathrm{~cm}$ in length) were used for both the inflow and outflow tubes. A roller pump was not used, and only the fetal heart pumped the blood. Both the fetus and the circuit were submerged and warmed in a saline bath kept at $39^{\circ} \mathrm{C}$. Pure oxygen was supplied to the membranous oxygenator at flow rates below $1.01 / \mathrm{min}$. The circuit was primed with $60 \mathrm{ml}$ of heparinized maternal blood. 


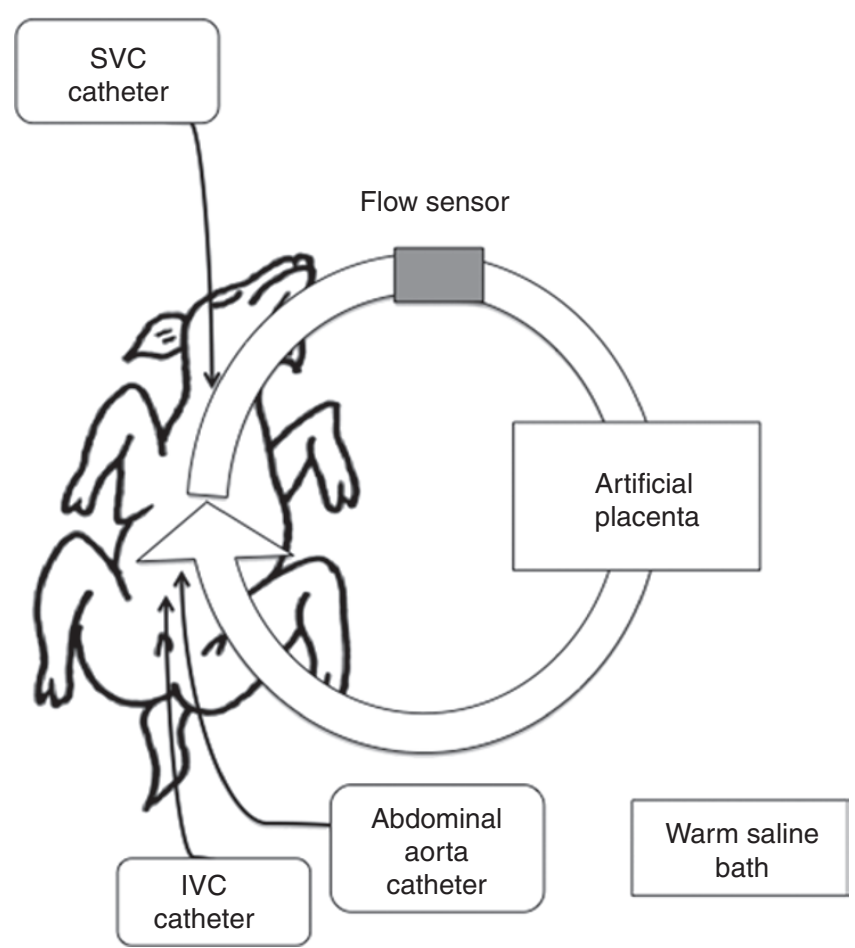

Figure 3. Schema of the artificial placenta. A roller pump was not used. Both the fetus and the circuit were submerged and warmed in a saline bath kept at $39^{\circ} \mathrm{C}$. IVC, inferior vena cava; SVC, superior vena cava.

\section{Data Acquisition}

The fetal heart rate, MAP, central venous pressure, and amniotic pressure were continuously monitored using a polygraph. Pressure values obtained before birth were corrected according to amniotic fluid pressure. Hemoglobin levels, $\mathrm{pH}$, base excess, and $\mathrm{P}_{\mathrm{O}}, \mathrm{P}_{\mathrm{CO} 2}$ (Blood Gas System 860; Bayer Medical, Sudbury, UK), and $\mathrm{O}_{2}$ content (OSM3 hemoximeter; Radiometer Medical, Copenhagen, Denmark) were measured in blood samples $(0.5 \mathrm{ml})$ obtained from the fetal abdominal aorta at least once every $4 \mathrm{~h}$. Blood gas data obtained before and after birth were corrected using the maternal rectal temperature and the fetal central temperature, respectively. The activated clotting times were monitored using a Hemochron 401 (ITC Nexus Dx, Edison, NJ) and were maintained for more than $180 \mathrm{~s}$. The extracorporeal circulatory blood flow $(\mathrm{ml} / \mathrm{min})$ was determined using an electromagnetic blood flowmeter (Transonic 400-Series; Transonic Systems, Ithaca, NY), which was attached to the arterial position of the blood circuit. The performance of the membranous oxygenator was calculated at 1-6 and 6-24h after birth. Each gas transfer rate was calculated using the formula below.

Gas transfer rate $(\mathrm{ml} / \mathrm{min})=\Delta \mathrm{C} \times \mathrm{W}$

$\Delta \mathrm{C}=$ concentration change in blood gas $(\mathrm{ml}($ gas $) / \mathrm{ml}$ (blood)

$\mathrm{W}=$ quantity of blood flow $(\mathrm{ml} / \mathrm{min})$

\section{Tissue Blood Flow Measurements}

In this study, colored microspheres (Dye-Trak; Triton Technology, San Diego, CA) were used to determine tissue blood flow (17). The microspheres were injected over a period of $20 \mathrm{~s}$ so that the systemic hemodynamics remained unchanged during the entire injection time. Starting $10 \mathrm{~s}$ before the injection of microspheres and continuing for $90 \mathrm{~s}$ after the injection was completed, a reference blood sample was simultaneously withdrawn from the abdominal artery at a rate of $2 \mathrm{ml} / \mathrm{min}$ using a precision pump (KD Scientific, Holliston, MA). The cerebral gray matter, cerebral white matter, medulla oblongata, left heart ventricle, right heart ventricle, left lung, right lung, adrenal gland, liver, and kidney were carefully dissected free of the adherent fat or connective tissue after fetal death, and the tissues were cut into small samples and weighed. The sample weights ranged from $\sim 1$ to 3 grams. The trapped colored microspheres in each organ and the reference blood samples were quantified according to their dye content using spectrophotometry. After the digestion of tissue and blood samples using a strong alkaline solution, the microspheres were harvested using a polyester filter. The dye was recovered from the microspheres by adding $300 \mu \mathrm{l}$ of Cellosolve acetate. A spectrophotometric analysis of the mixed dye solutions was performed using a spectrophotometer (U-2810; Hitachi, Tokyo, Japan). Tissue blood flow was calculated using the following equation:

Tissue blood flow $\left(\mathrm{ml} \cdot \mathrm{min}^{-1} \cdot \mathrm{g}^{-1}\right)=\mathrm{A}_{\mathrm{s}} \cdot \mathrm{V}_{\mathrm{ref}} \cdot \mathrm{A}_{\mathrm{ref}}{ }^{-1} \cdot \mathrm{W}_{\mathrm{s}}^{-1}$

Where $A_{s}$ is the absorbance of the tissue sample, $V_{\text {ref }}$ is the reference blood flow, $\mathrm{A}_{\text {ref }}$ is the absorbance of reference blood sample, and $\mathrm{W}_{\mathrm{s}}$ is the weight of the tissue sample.

\section{Statistical Analysis}

All values are expressed as mean \pm SEM. We used SPSS for Windows for the statistical analysis. Student's $t$-test was used to compare the values of Reoma's experiments (6) with those of our study. The Wilcoxon rank sum test was used to compare the values for organ blood flow obtained after birth with those obtained before birth. The Wilcoxon rank sum test was also used to compare the gas transfer rates obtained between 1 and $6 \mathrm{~h}$ after birth with those obtained between 6 and $24 \mathrm{~h}$ after birth. The Spearman correlation coefficient was calculated to assess the relationships among circuit blood flow, $\mathrm{MAP}, \mathrm{O}_{2}$ content, $\mathrm{P}_{\mathrm{CO} 2}$, and blood lactate level. Spearman correlation coefficients $>0.7$ were regarded as indicating an apparent correlation, and scatter diagrams of the correlated parameters are shown in Figure 2. All probability values $<0.05$ were considered significant.

\section{STATEMENT OF FINANCIAL SUPPORT}

This study was supported by a Grant-in-Aid for Scientific Research from the Ministry of Education, Culture, Sports, Science and Technology, Tokyo, Japan (grants 19790755, 21791030, and 22791026) and the Ogyaa Donation Foundation of the Japan Association of Obstetricians and Gynecologists.

\section{ACKNOWLEDGMENTS}

The authors gratefully thank Keisuke Sanbongi and Tatsuya Watanabe for technical assistance. The abstract of this article was presented at the Fetal and Neonatal Physiological Society 2011 Meeting, Palm Cove, Australia, 10-13 July 2011.

\section{REFERENCES}

1. Callaghan JC, Maynes EA, Hug HR. Studies on lambs of the development of an artificial placenta. Review of nine long-term survivors of extracorporeal circulation maintained in a fluid medium. Can J Surg 1965;8:208-13.

2. Alexander DP, Britton HG, Nixon DA. Maintenance of sheep fetuses by an extracorporeal circuit for periods up to 24 hours. Am J Obstet Gynecol 1968;102:969-75.

3. Zapol WM, Kolobow T, Pierce JE, Vurek GG, Bowman RL. Artificial placenta: two days of total extrauterine support of the isolated premature lamb fetus. Science 1969;166:617-8.

4. Unno N, Kuwabara Y, Okai T, et al. Development of an artificial placenta: survival of isolated goat fetuses for three weeks with umbilical arteriovenous extracorporeal membrane oxygenation. Artif Organs 1993;17:996-1003.

5. Sakata M, Hisano K, Okada M, Yasufuku M. A new artificial placenta with a centrifugal pump: long-term total extrauterine support of goat fetuses. J Thorac Cardiovasc Surg 1998;115:1023-31.

6. Reoma JL, Rojas A, Kim AC, et al. Development of an artificial placenta I pumpless arterio-venous extracorporeal life support in a neonatal sheep model. J Pediatr Surg 2009;44:53-9.

7. Funakubo A, Sakuma I, Fukui Y, Kawamura T. Development of a compact extracorporeal membrane oxygenation (ECMO) system. Artif Organs 1991;15:56-9.

8. Funakubo A, Higami T, Sakuma I, et al. Development of a membrane oxygenator for ECMO using a novel fine silicone hollow fiber. ASAIO J 1996;42:M837-40. 


\section{Articles | Miuraet al.}

9. Faber JJ, Green TJ. Foetal placental blood flow in the lamb. J Physiol (Lond) 1972;223:375-93

10. Creasy RK, Drost M, Green MV, Morris JA. Determination of fetal, placental and neonatal blood volumes in the sheep. Circ Res 1970;27:487-94.

11. Assad RS, Lee FY, Hanley FL. Placental compliance during fetal extracorporeal circulation. J Appl Physiol 2001;90:1882-6.

12. Parisi VM, Walsh SW. Fetal vascular responses to prostacyclin. Am J Obstet Gynecol 1989;160:871-6; discussion 876-8.

13. Saito M, Matsuda T, Okuyama K, et al. Effect of intrauterine inflammation on fetal cerebral hemodynamics and white-matter injury in chronically instrumented fetal sheep. Am J Obstet Gynecol 2009;200:663.e1-11.

14. Watanabe T, Matsuda T, Hanita T, et al. Induction of necrotizing funisitis by fetal administration of intravenous granulocyte-colony stimulating factor and intra-amniotic endotoxin in premature fetal sheep. Pediatr Res 2007;62:670-3.

15. Kusaka T, Matsuda T, Okuyama K, et al. Analyses of factors contributing to vulnerability to antenatal periventricular leukomalacia induced by hemorrhagic hypotension in chronically instrumented fetal sheep. Pediatr Res 2002;51:20-4.

16. Matsuda $\mathrm{T}$, Okuyama $\mathrm{K}$, Cho $\mathrm{K}$, et al. Induction of antenatal periventricular leukomalacia by hemorrhagic hypotension in the chronically instrumented fetal sheep. Am J Obstet Gynecol 1999;181: 725-30.

17. Kowallik P, Schulz R, Guth BD, et al. Measurement of regional myocardial blood flow with multiple colored microspheres. Circulation 1991;83:974-82. 